\title{
Análise Espectral de um Método Pseudo Espectral para Propagação de Onda com Condições de Fronteira Transparentes
}

P.C. CALEGARI ${ }^{1}$, F.S.V. BAZÁN ${ }^{2}$, Departamento de Matemática, Universidade Federal de Santa Catarina, 88040-900 Florianópolis, SC, Brasil.

Resumo. Apresentamos uma análise espectral de um método para a simulação numérica de propagação de ondas unidimensionais, proposto recentemente por Jackiewicz e Renaut em [4]. Os resultados incluem uma fórmula fechada para o auto sistema do operador associado ao problema, um resultado teórico que corrige uma conclusão errônea desses autores e um método numérico para calcular soluções aproximadas. Além disso, destacamos a importância da análise do pseudo espectro do operador do modelo contínuo, assim como o pseudo espectro das matrizes dos modelos discretos correspondentes.

\section{Introdução}

Problemas de propagação de ondas em domínios extensos ou provavelmente não limitados, são abordados na prática restringindo o domínio para uma região delimitada por fronteiras artificiais, na qual constroem-se soluções aproximadas usando métodos numéricos. Um aspecto crucial do processo é a escolha de condições de fronteira que atenuem as reflexões espúrias da solução numérica, que aparecem como conseqüência do truncamento do domínio original através de fronteiras artificiais. Condições desse tipo são chamadas de condições de fronteira absorventes [2]. As condições de fronteira que evitam completamente as reflexões, são ditas transparentes $[3,4]$.

Neste trabalho continuamos uma investigação de Renaut [7], e Jackiewicz e Renaut [4], sobre a estabilidade de um método pseudo espectral de Chebyshev para a propagação da onda unidimensional, restrita ao intervalo $[0,1]$ e com condições de fronteira absorventes. Para tanto, usamos uma reformulação da equação da onda na forma $v_{t}=\mathcal{A} v[2,4,6,12]$, onde $\mathcal{A}$ é um operador diferencial que não depende de $t$ e definido em um espaço de Hilbert apropriado. Primeiro o método discretiza o operador diferencial produzindo um sistema de EDO's da forma $\dot{v}_{N}=A_{N} v_{N}$, onde $A_{N}$ é uma matriz associada a discretização das derivadas na variável espacial através de diferenciação de Chebyshev [10], e em seguida constrói soluções numéricas resolvendo o sistema de EDO's através do método de Runge-Kutta de quarta ordem.

\footnotetext{
${ }^{1}$ priscila@mtm.ufsc.br; Mestrado em Matemática e Computação Científica

${ }^{2}$ fermin@mtm.ufsc.br; CNPq grant 201407/03-5(NV)
} 
Enfatizamos que a estabilidade do sistema semi-discreto depende fortemente da não normalidade do operador $\mathcal{A}$ e que análises baseadas somente no espectro de $A_{N}$ podem levar a conclusões errôneas sobre a estabilidade das soluções numéricas: é conhecido que para estabilidade das soluções numéricas do sistema semi discreto com tamanho de passo $\Delta t$ é suficiente que o pseudo espectro da matriz $\Delta t A_{N}$ pertença à região de estabilidade absoluta do método $[5,6]$, sendo a convergência da solução, garantida pelo teorema da equivalência de Lax $[5,6,9]$.

O trabalho apresenta duas contribuições. Após uma reformulação do problema descrita na Seção 2, a primeira contribuição vem na Seção 3 na qual apresentamos fórmulas fechadas para o auto sistema do operador $\mathcal{A}$ que generalizam resultados prévios de Driscoll e Trefethen [2], que consideram uma condição de fronteira Dirichlet na esquerda do domínio e outra absorvente na direita. Uma análise do pseudo espectro do operador também é incluída. A segunda contribuição vem na Seção 4, na qual primeiro descrevemos o método de Jackiewicz e Renaut, e a seguir mostramos teórica e numericamente que um resultado desses autores é incorreto. Incluímos também uma abordagem numérica que apresenta vantagens em relação a proposta desses autores. Na Seção 5, apresentamos algumas considerações finais.

\section{Modelo Contínuo}

Nesta seção, apresentamos algumas características físicas do problema e a reformulação do problema em um sistema autônomo que usaremos ao longo do trabalho. Vamos considerar a equação da onda, com velocidade constante $c$ :

$$
\begin{aligned}
& u_{t t}-c^{2} u_{x x}=0, \quad x \in \mathbb{R}, t>0 \\
& u(x, 0)=f(x), \quad u_{t}(x, 0)=0
\end{aligned}
$$

onde $u(x, 0)$ e $u_{t}(x, 0)$ são condições iniciais do problema. A solução de (2.1) é dada pela fórmula de D'Alembert: $u(x, t)=\frac{1}{2}(f(x+c t)+f(x-c t))$. Verifica-se que enquanto o primeiro termo $(x, t) \rightarrow \frac{1}{2} f(x+c t)$, se propaga para a esquerda e satisfaz

$$
\left(\frac{\partial}{\partial t}-c \frac{\partial}{\partial x}\right) \frac{1}{2} f(x+c t)=0,
$$

o segundo termo $(x, t) \longrightarrow \frac{1}{2} f(x-c t)$, se propaga para a direita satisfazendo,

$$
\left(\frac{\partial}{\partial t}+c \frac{\partial}{\partial x}\right) \frac{1}{2} f(x-c t)=0 .
$$

O comportamento da solução segundo (2.2) e (2.3) sugere que a solução de (2.1) restrita ao intervalo $[0,1]$ pode ser encontrada adicionando-se a $(2.1)$ as condições de fronteira

$$
\begin{aligned}
& u_{t}(0, t)-c u_{x}(0, t)=0 \\
& u_{t}(1, t)+c u_{x}(1, t)=0
\end{aligned} .
$$

As condições de fronteira (2.4) são completamente transparentes, isto é, permitem que as ondas atravessem as fronteiras artificiais eliminando completamente o fenômeno de reflexão $[3,4,1]$. A Tabela 1 apresenta diferentes condições de 
fronteira (para $x=0$ ) usadas freqüentemente em conexão com a equação da onda e seus respectivos coeficientes de reflexão. As condições de fronteira de Dirichlet e Neumann são perfeitamente reflexivas, sendo que na primeira a onda refletida é invertida. Note que $\delta>0$ define uma família de condições de fronteira absorventes e o caso $\delta<0$ introduz um termo de fonte, impondo energia ao sistema $[3,1]$.

\begin{tabular}{|c|c|c|}
\hline \multicolumn{2}{|c|}{ Condições de fronteira } & Coeficiente de reflexão \\
\hline \hline Dirichlet & $u=0$ & $R=-1$ \\
\hline Neumann & $u_{x}=0$ & $R=1$ \\
\hline Absorventes & $\delta u_{t}-c u_{x}=0$ & $R=\frac{1-\delta}{1+\delta}$ \\
\hline Transparentes & $u_{t}-c u_{x}=0$ & $R=0$ \\
\hline
\end{tabular}

Tabela 1: Condições de fronteira $\times$ Coeficiente de reflexão.

Para nossa análise precisamos reformular o problema original. Utilizando as variáveis auxiliares $v_{1}=u_{t}$ e $v_{2}=u_{x}$ obtemos

$$
\frac{\partial v_{1}}{\partial t}=c^{2} \frac{\partial v_{2}}{\partial x} \quad \text { e } \quad \frac{\partial v_{2}}{\partial t}=\frac{\partial v_{1}}{\partial x}
$$

o que nos leva ao sistema da forma

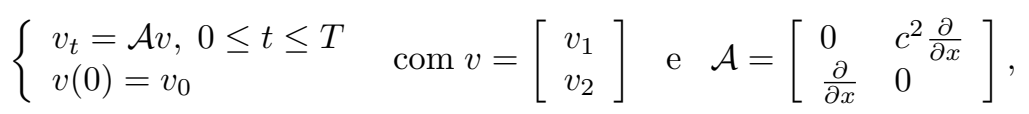

sendo $\mathcal{A}$ um operador linear definido em $\mathcal{A}: \mathcal{D} \mapsto \mathcal{H}$, onde $\mathcal{H}=L^{2}(0,1) \times L^{2}(0,1)$ e $\mathcal{D}$ é um subespaço denso em $\mathcal{H}$ formado por funções absolutamente contínuas $v=\left[\begin{array}{ll}v_{1} & v_{2}\end{array}\right]^{T}$, satisfazendo as condições de fronteira absorventes

$$
\begin{aligned}
& v_{1}(0, t)-c_{1} v_{2}(0, t)=0 \\
& v_{1}(1, t)+c_{2} v_{2}(1, t)=0
\end{aligned},
$$

com $c_{1}$ e $c_{2}$ constantes positivas. Note que (2.6) torna-se (2.4) quando $c_{1}=c_{2}=c$. Observe também que um problema do tipo (2.5), cuja solução é $v(t)=\exp (t \mathcal{A}) v(0)$, é bem posto se e somente se para cada $t$ existe uma constante $C_{1}>0$ tal que $\|\exp (t \mathcal{A})\| \leq C_{1}$ para alguma norma $\|\cdot\|$ convenientemente definida, e em particular, se todos os autovalores de $\mathcal{A}$ tem parte real negativa, $\|\exp (t \mathcal{A})\| \rightarrow 0$ quando $t \rightarrow \infty[9]$.

\section{Espectro e Pseudo Espectro}

A discussão acima mostra que faz-se necessária uma análise espectral do operador $\mathcal{A}$, tanto para $c_{1}, c_{2}$ arbitrárias, bem como para $c_{1}, c_{2}$ tendendo a $c$. A seguinte proposição descreve o auto sistema de $\mathcal{A}$.

Proposição 3.1. Assuma que $c_{1}<c$ e $c_{2}<c$. Então os autovalores de $\mathcal{A}$ são

$$
\lambda_{n}=\frac{c}{2} \ln \left(\frac{\left(c-c_{1}\right)\left(c-c_{2}\right)}{\left(c+c_{1}\right)\left(c+c_{2}\right)}\right)+c n \pi i, \quad \forall n \in \mathbb{Z} .
$$


Demonstração. Seja $\lambda$ um autovalor de $\mathcal{A}$ e $v=\left[\begin{array}{ll}v_{1} & v_{2}\end{array}\right]^{T}$ a autofunção correspondente. A equação $\mathcal{A} v=\lambda v$ exige que

$$
\frac{\partial^{2} v_{1}}{\partial x^{2}}-\frac{\lambda^{2}}{c^{2}} v_{1}=0, \quad \text { e } \quad \frac{\partial^{2} v_{2}}{\partial x^{2}}-\frac{\lambda^{2}}{c^{2}} v_{2}=0,
$$

e dessas equações segue que as componentes da autofunção $v$ devem ser da forma

$$
v_{1}(x)=A_{1} \exp \left(\frac{\lambda x}{c}\right)+A_{2} \exp \left(-\frac{\lambda x}{c}\right), \text { e } v_{2}(x)=B_{1} \exp \left(\frac{\lambda x}{c}\right)+B_{2} \exp \left(-\frac{\lambda x}{c}\right)
$$

com $A_{i}$ e $B_{i}$ a serem determinadas. Agora note que a primeira condição de fronteira em (2.6) implica que $A_{1}=c B_{1}, A_{2}=-c B_{2}$ e $B_{2}=\left(\frac{c-c_{1}}{c+c_{1}}\right) B_{1}$. A escolha $B_{1}=\frac{1}{2}$ implica então que

$$
v_{1}(x)=\frac{c}{c+1}\left(c \sinh \frac{\lambda x}{c}+c_{1} \cosh \frac{\lambda x}{c}\right), v_{2}(x)=\frac{1}{c+c_{1}}\left(c \cosh \frac{\lambda x}{c}+c_{1} \sinh \frac{\lambda x}{c}\right)
$$

e a segunda condição de fronteira em (2.6) mostra que

$$
\exp \left(2 \frac{\lambda}{c}\right)=\frac{\left(c-c_{1}\right)\left(c-c_{2}\right)}{\left(c+c_{1}\right)\left(c+c_{2}\right)}
$$

o qual verifica (3.1).

Observação 1. Podemos também transformar o problema (2.1) em um sistema de equações diferenciais ordinárias, definido da seguinte maneira,

$$
\left\{\begin{array}{l}
w_{t}=\mathcal{B} w \\
w(0)=w_{0}
\end{array}, \quad \operatorname{com} \mathcal{B}=\left[\begin{array}{ll}
0 & I \\
c^{2} \frac{\partial^{2}}{\partial x^{2}} & 0
\end{array}\right], \quad \text { e } w=\left[\begin{array}{c}
u \\
u_{t}
\end{array}\right] .\right.
$$

A partir desta formulação, os autovalores de $\mathcal{B}$ são dados por (3.1).

Observação 2. O espectro do operador $\mathcal{A}, \wedge(\mathcal{A})$, é vazio quando $c_{1}=c_{2}=c$. Isto pode ser explicado intuitivamento notando que, como as autofunções devem ser da forma (3.3) e já que elas dependem de $\lambda$ satisfazendo (3.4), conclui-se que não existem autofunções no caso $c_{1}=c_{2}=c$. Uma demonstração rigorosa deste resultado foge do escopo deste trabalho; ela requer uma análise do operador resolvente $(z I-\mathcal{A})^{-1}, z \in \mathbb{C} ;$ o leitor interessado pode se direcionar a Driscoll e Trefethen [2].

Da observação 2 segue que análises baseadas somente no espectro de $\mathcal{A}$ podem não ser suficientes para informar o comportamento do sistema no decorrer do tempo. Em casos como este, faz-se necessária a análise do pseudo espectro do operador, conforme segue.

Seja $\mathcal{X}$ um espaço de Banach munido de uma norma $\|\cdot\|$, e $\mathcal{A}: \mathcal{X} \mapsto \mathcal{X}$ um operador linear fechado. Resumidamente, o pseudo espectro de um operador $\mathcal{A}$ é o conjunto de todos os autovalores do operador $\widetilde{\mathcal{A}}=\mathcal{A}+E$, com $\|E\|<\epsilon$. A definição 3.1 caracteriza formalmente o pseudo espectro de operadores.

Definição 3.1. Dado $\epsilon>0$, o $\epsilon$-pseudo espectro de $\mathcal{A}, \wedge_{\epsilon}(\mathcal{A})$, é o conjunto dos números $z \in \mathcal{C}$, definido equivalentemente por uma das seguintes condições: 
i. $\left\|(z \mathcal{I}-\mathcal{A})^{-1}\right\| \geq \epsilon^{-1}, \mathcal{I}$ é o operador identidade.

ii. $z \in \wedge(\mathcal{A}+E)$ para algum operador $E$, com $\|E\|<\epsilon$.

iii. $z \in \wedge(\mathcal{A})$ ou $\|(z \mathcal{I}-\mathcal{A}) u\|<\epsilon$ para algum $u \in D(\mathcal{A})$ com $\|u\|=1$.

Se $\|(z \mathcal{I}-\mathcal{A}) u\|<\epsilon$ como em (iii.), então z é um $\epsilon$-pseudo autovalor de $\mathcal{A}$ e u o $\epsilon-$ pseudo autovetor (ou $\epsilon$-pseudo autofunção) correspondente.

Se $\mathcal{A}$ é uma matriz ou operador normal, o $\epsilon$-pseudo espectro $\wedge_{\epsilon}(\mathcal{A})$ é formado pela união de bolas de raio $\epsilon$ centradas nos pontos do espectro de $\mathcal{A}$. O que implica que os autovalores de $\mathcal{A}$ são insensíveis a pequenas perturbações. Se $\mathcal{A}$ está longe da normalidade, $\wedge_{\epsilon}(\mathcal{A})$ pode ser muito grande e ter um contorno muito diferente: quanto maior o pseudo espectro, maior a não normalidade do operador [11].
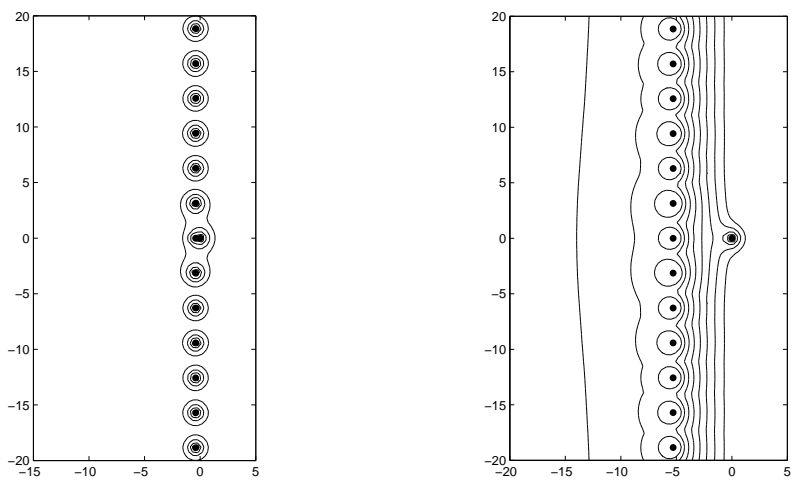

Figura 1: Fronteiras do $\epsilon$-pseudo espectro de $\mathcal{A}$ para $c_{1}=c_{2}=0.2$ e $c_{1}=c_{2}=0.99$, esquerda e direita respectivamente, $\operatorname{com} \epsilon=10^{-1.4}, 10^{-1.2}, \ldots, 10^{0}$. Os autovalores de $\mathcal{A}$ são denotados por •

O pseudo espectro de $\mathcal{A}$ definido em (2.5) é apresentado nas Figuras 1 e 2 para o caso em que a constante da equação da onda é $c=1$ e algumas escolhas de $c_{1}, c_{2}$. Observe que a medida que $c_{1}, c_{2} \rightarrow c$, na figura 1 , o pseudo espectro tornase mais amplo e a não normalidade do operador $\mathcal{A}$ cresce, i.e., os autovalores do operador tornam-se mal condicionados. Já a Figura 2, apresenta o pseudo espectro do operador para $c=c_{1}=c_{2}$. Nesse caso, os $\epsilon$-pseudo espectros são os semi-planos à esquerda das fronteiras. Os pseudo espectros foram calculados através de um modelo semi discreto de dimensão suficientemente grande, a ser apresentado na próxima seção ${ }^{3}$.

\section{Problema Semi Discreto}

O objetivo da seção é analisar algumas versões semi discretas para a problema (2.1) com condições de fronteira absorventes (2.6). Todos os modelos provém de aplicar

\footnotetext{
${ }^{3}$ Todos os gráficos sobre pseudo espectro apresentados nesse trabalho, foram construídos usando o pacote EigTool [13].
} 


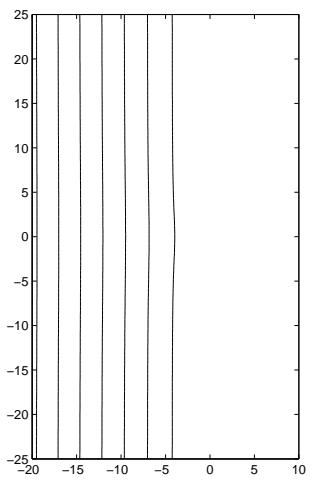

Figura 2: Fronteiras do $\epsilon$-pseudo espectro de $\mathcal{A}$ para $c_{1}=c_{2}=c=1$, com $\epsilon=$ $10^{-7}, 10^{-6}, \ldots, 10^{-1}$.

o método pseudo espectral de Chebyshev com pontos de Chebyshev-Gauss-Lobatto como pontos colocação: $x_{0}=0, \ldots, x_{N}=1$. Um aspecto fundamental do método é o uso da matriz de diferenciação de Chebyshev denotada por $D$ e cujas linhas e colunas são denotadas respectivamente por $d_{i}$ e $l_{i}^{T}, 0 \leq i \leq N$. Detalhes sobre diferenciação discreta de Chebyshev podem ser vistos em [10].

O método de Jackiewicz e Renaut [4] provém do modelo contínuo (2.5), no qual são apresentadas duas formas de incorporar as condições de fronteira, sendo $D_{1}=\left[d_{1}, \ldots, d_{N-1}\right]$ e $D_{2}=\left[l_{1}, \ldots, l_{N-1}\right]^{T}$. A primeira vem da observação de que se fazemos

$$
v_{2}\left(x_{0}, t\right)=v_{1}\left(x_{0}, t\right) / c_{1}, \text { e } \quad v_{2}\left(x_{N}, t\right)=-v_{1}\left(x_{N}, t\right) / c_{2},
$$

e se $w_{1} \approx\left[v_{1}\left(x_{0}, t\right), \ldots, v_{1}\left(x_{N}, t\right), v_{2}\left(x_{1}, t\right), \ldots, v_{2}\left(x_{N-1}, t\right)\right]^{T}$, i.e., $w_{1}$, é o vetor de soluções numéricas nos pontos da malha, então o modelo semi discreto é:

$$
\dot{w}_{1}=A_{1} w_{1}, \quad \text { com } A_{1}=\left[\begin{array}{cc}
C & c^{2} D_{1} \\
D_{2} & O_{(N-1) \times(N-1)}
\end{array}\right],
$$

mas, se escolhemos

$$
v_{1}\left(x_{0}, t\right)=c_{1} v_{2}\left(x_{0}, t\right), \text { e } v_{1}\left(x_{N}, t\right)=-c_{2} v_{2}\left(x_{N}, t\right)
$$

e $w_{2} \approx\left[v_{1}\left(x_{1}, t\right), \ldots, v_{1}\left(x_{N-1}, t\right), v_{2}\left(x_{0}, t\right), \ldots, v_{2}\left(x_{N}, t\right)\right]^{T}$, o modelo discreto é

$$
\dot{w}_{2}=A_{2} w_{2}, \quad \text { com } A_{2}=\left[\begin{array}{cc}
O_{(N-1) \times(N-1)} & c^{2} D_{2} \\
D_{1} & C
\end{array}\right] .
$$

Em ambos os modelos (4.1) e (4.2), $C=\left[c d_{0}, 0, \ldots, 0,-c d_{N}\right]$ pois $c_{1}=c_{2}=c$, os 0 's representam vetores coluna nulos, $(N+1) \times 1$ e as matrizes $A_{1}$ e $A_{2}$ possuem dimensão $2 N$.

A estabilidade das soluções numéricas dos modelos pelo método de Runge-Kutta de quarta ordem foi analisada em [4] onde é determinado numericamente o tamanho de passo $\Delta t=0.004$, que garante a estabilidade. A determinação do $\Delta t$ foi 
feito a partir do espectro e pseudo espectro de $A_{1}$ e $A_{2}$. A conclusão foi que as características de estabilidade das soluções numéricas de ambas as formulações são muito semelhantes, apesar das matrizes terem espectros diferentes (com $A_{1}$ tendo autovalores no semi plano direito). Nós concordamos com a primeira afirmação, não com a segunda. A seguinte proposição mostra que essa afirmação é incorreta.

Proposição 4.1. As matrizes $A_{1}$ e $A_{2}$ são semelhantes.

Demonstração. Defina $S=\left[\begin{array}{cc}O_{(N-1) \times(N+1)} & c^{2} I_{N-1} \\ I_{N+1} & O_{(N+1) \times(N-1)}\end{array}\right]$. Segue facilmente que $S^{-1}=\left[\begin{array}{cc}0_{(N+1) \times(N-1)} & I_{N+1} \\ \frac{1}{c^{2}} I_{N-1} & O_{(N-1) \times(N+1)}\end{array}\right]$ e daí que $A_{1}=S^{-1} A_{2} S$, como requerido.

Note que $c=1$ produz uma transformação de semelhança que é ortogonal. Nesse caso o pseudo espectro de ambas as matrizes coincidem. Se $c \approx 1, S$ é quase normal e nesse caso os pseudo espectros de $A_{1} \mathrm{e} A_{2}$ não devem diferir muito por causa da quase normalidade da transformação de semelhança. Ilustramos este fato na Figura 3 para $c=0.5$ e $N=30$.
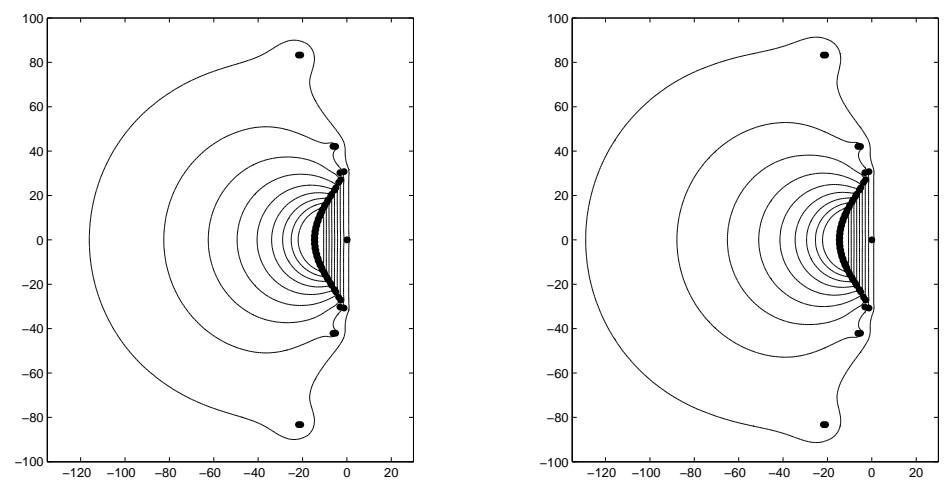

Figura 3: Pseudo espectro de $A_{1}$ e $A_{2}$, esquerda e direita respectivamente, com $\epsilon=10^{-8}, 10^{-7}, \ldots, 10^{0}$.

\subsection{Método Proposto}

Visto que a abordagem de Jackiewicz e Renaut não calcula deslocamento e sim aproximações para $u_{t}$ e $u_{x}$ (que torna-se importante quando procura-se a energia do sistema), apresentamos uma formulação semi discreta que permite o cálculo direto da solução $u$ a cada passo do tempo e que provém da reformulação (3.5) do problema. Para tal, note que a aproximação da derivada espacial de segunda ordem via matriz diferenciação de Chebyshev é $D^{2}=D D=d_{0} l_{0}^{T}+\ldots+d_{N} l_{N}^{T}$. Se $\bar{u}=\left[u\left(x_{0}, t\right), \ldots, u\left(x_{N}, t\right)\right]^{T}$, em cada ponto da malha podemos escrever

$$
\bar{u}_{t t} \approx c^{2}\left[d_{0} l_{0}^{T} \bar{u}+d_{1} l_{1}^{T} \bar{u}+\cdots+d_{N-1} l_{N-1}^{T} \bar{u}+c_{N} d_{N}^{T} \bar{u}\right]
$$



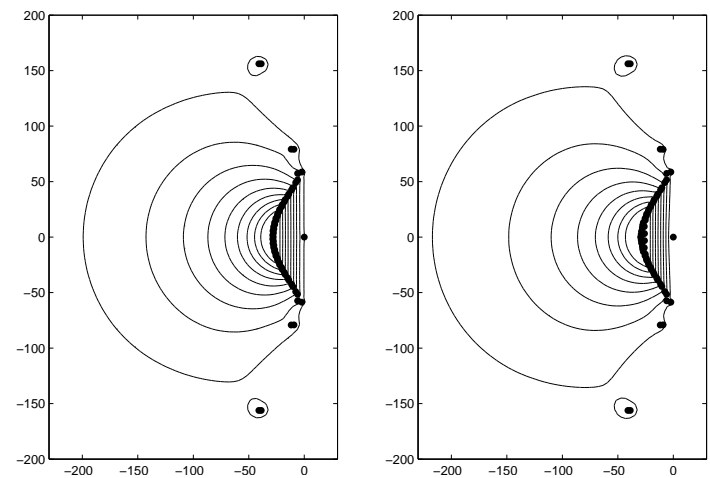

Figura 4: Fronteiras do $\epsilon$-pseudo espectro da matrizes $A_{1}$ e $L_{N}$, esquerda e direita respectivamente, $\operatorname{com} c=1, N=30$ e $\epsilon=10^{-8}, 10^{-7}, \ldots, 10^{0}$ (para $A_{1}$ ) e $\epsilon=$ $10^{-8}, 10^{-7}, \ldots, 10^{-2}\left(\right.$ para $\left.L_{N}\right)$.

Mas, já que $l_{0}^{T} \bar{u} \approx u_{x}\left(x_{0}, t\right)$ e $l_{N}^{T} \bar{u} \approx u_{x}\left(x_{N}, t\right)$, das condições de fronteira (2.4) temos que $u_{x}\left(x_{0}, t\right)=(1 / c) u_{t}\left(x_{0}, t\right)$ e $u_{x}\left(x_{N}, t\right)=(1 / c) u_{t}\left(x_{N}, t\right)$. Portanto, segue que (4.3) pode ser reescrito como um sistema de EDO's de segunda ordem da forma

$$
\bar{u}_{t t}-C \bar{u}_{t}-c^{2} \widetilde{D} \bar{u}=0
$$

em que $\widetilde{D}=d_{1} l_{1}^{T}+\ldots+d_{N-1} l_{N-1}^{T}$ com $C$ como em (4.2). Considerando as condições iniciais $u(x, 0)=f(x), u_{t}(x, 0)=0$, e transformando o sistema acima para um sistema de primeira ordem, obtemos o problema de valor inicial

$$
\left\{\begin{array}{l}
\dot{\bar{v}}=L_{N} \bar{v} \\
\bar{v}(0)=\left[f\left(x_{0}\right), \ldots, f\left(x_{N}\right), 0_{1 \times N}\right]^{T} \quad, \quad L_{N}=\left[\begin{array}{ll}
O & I \\
c^{2} \widetilde{D} & C
\end{array}\right]
\end{array}\right.
$$

em que $O$ e $I$ representam, respectivamente, as matrizes nula e identidade de ordem $N+1$, e $\bar{v}=\left[\begin{array}{ll}\bar{u} & \bar{u}_{t}\end{array}\right]^{T}$.

Nossa proposta consiste em resolver o sistema semidiscreto (4.5) através do método de Runge-Kutta de quarta ordem. Note que enquanto $L_{N}$ é de ordem $2 N+2$, a ordem de $A_{1}$ e $A_{2}$ é $2 N$. A seguinte proposição, cuja demonstração é encontrada em [1], mostra que o espectro de $L_{N}$ contém basicamente a mesma informação do que o espectro de $A_{1}$.

Proposição 4.2. Assuma que $c_{1}=c_{2}=c$. Então o espectro da matriz $L_{N}$ definida em (4.5) satisfaz

$$
\wedge\left(L_{N}\right)=\wedge\left(A_{1}\right) \cup\{0\} .
$$

A Figura, 4 mostra que o pseudo espectro da matriz $L_{N}$ não difere muito do pseudo espectro de $A_{1}$ ou de $A_{2}$. Disso conclui-se que as características de estabilidade das soluções numéricas obtidas a partir de nossa proposta não devem deteriorar. 

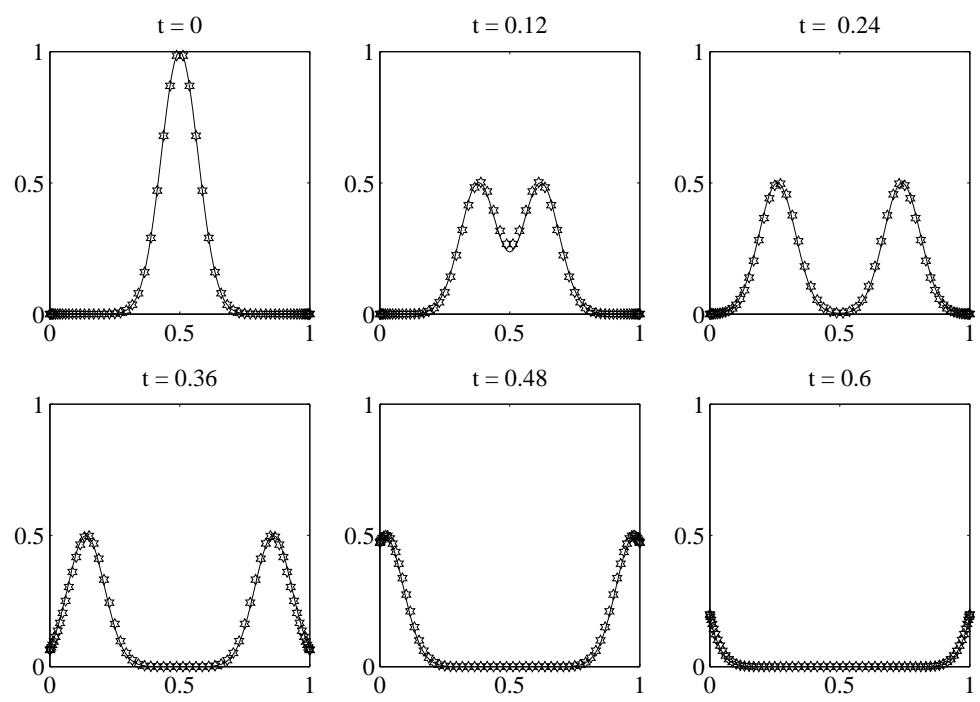

Figura 5: Solução exata $u$ - e solução aproximada $v \cdot$, no tempo $t=0, t=0.15$, $t=0.45$ e $t=0.6$, respectivamente.

\subsection{Exemplo Numérico}

Para testar o método proposto resolvemos o problema com $c=c_{2}=c_{1}=1$, condição inicial $u(x, 0)=f(x) \equiv \exp \left(-100(x-0.5)^{2}\right), 0 \leq x \leq 1$, e usamos o mesmo tamanho de passo $\Delta t$ determinado por Jackiewicz e Reanut [4]. Os resultados são apresentados na Figura 5. Note que neste caso, conforme esperado, as reflexões da solução são completamente eliminadas.

\section{Considerações Finais}

Temos apresentado uma análise espectral de um método para simular a propagação de ondas unidimensionais. Os resultados mais importantes são a descrição de fórmulas fechadas para o auto sistema de um operador associado ao problema, e uma revisão de um método descrito em [4] que permitiu: a) mostrar a falsidade de uma conclusão nesse trabalho, e b) apresentar um método para o problema com praticamente as mesmas características de estabilidade daquele em [4]. A teoria apresentada e a qualidade dos resultados do método foram ilustrados através de simulações numéricas. Nosso próximo passo será estender esses resultados para o caso bi-dimensional. Faz-se necessária uma análise teórica mais profunda sobre a estabilidade do método de Runge Kutta associado ao sistema $\bar{u}_{t}=L_{N} \bar{u}$.

Abstract. We present a spectral analysis of a method for numerical simulation of $1 \mathrm{D}$ wave propagation recently proposed by Jackewicz and Renaut in [4]. The results include a closed formula for the eigensystem of the operator associated with the problem, a theoretical result that corrects a wrong conclusion in [4], and a 
proposal for determining a numerical solution. Numerical results are also included and the importance of a pseudospectral analysis in connection with stability issues is emphasized.

\section{Referências}

[1] P.C. Calegari, "Método Pseudo Espectral de Chebyshev para Problemas de Propagação de Ondas com Condições de Fronteira Absorventes", Dissertação de Mestrado em Matemática e Computação Científica, Universidade Federal de Santa Catarina, Florianópolis 2007.

[2] T.A. Driscol, L.N. Trefethen, Pseudospectra of the wave operator with an absorbing boundary, J. Comput. Appl. Math., 69 (1996), 125-142.

[3] L. Halpern, A. Rahmouni, "One Way Operators, Absorbing boundary conditions and domain decomposition for wave propagation", Modern Methods in Scientific Computing and Applications, 155-209. A. Bourliex et M.J.Gauder editeurs. Kluwer Academic Publishers, 2002.

[4] Z. Jackiewicz, R.A. Renaut, A note on stability of pseudospectral methods for wave propagations, J. Comput. Appl. Math., 143 (2002), 127-139.

[5] S.C. Reddy, L.N. Trefethen, Lax-stability of fully discrete spectral methods via stability regions and pseudo-eigenvalues, Comput. Meth Appl. Mech. Eng., 80 (1990), 147-164.

[6] S.C. Reddy, L.N. Trefethen, Stability of the method of lines, Num. Math., 62 (1992), 235-267.

[7] R. Renaut, Stability of a Chebyshev pseudoespectral solution of the wave equation with absorbing boundaries, J. Comput. Appl. Math., 87 (1997), 243-259.

[8] R.D. Richtmyer, K.W. Morton, "Difference Methods for Initial-value Problems", 2nd ed., John Wiley, New York, 1967.

[9] J.C. Strikwerda, "Finite Difference Schemes and Partial Differential Equations", Wadsworth \& Brooks, Califórnia, 1989.

[10] L.N. Trefethen, "Spectral Methods in Matlab", Society for Industrial and Applied Mathematics, Philadelphia, 2000.

[11] L.N. Trefethen, M. Embree, "Spectra e Pseudospectra - The behavior of Nonnormal Matrices o Operators", Princeton University Press, Princeton, 2005.

[12] K. Veselić, On linear vibrational systems with one-dimensional damping, Applicable Anal., 29 (1988), 1-18.

[13] T.G. Wright, "Matlab codes and graphical user interfaces for computation of pseudospectra", available at

http://www.comlab.ox.ac.uk/oucl/work/nick.trefethen 